\title{
Corresponsabilidad de la comunidad internacional en la explotación del bloque 430 ITT en el Parque Nacional Yasuní en Ecuador
}

por Diana Villacís Luna*

\section{- Resumen}

El presente artículo aborda las principales problemáticas surgidas a la raíz de la Iniciativa Yasuní ITT por la cual el Ecuador pretendía evitar la explotación del petróleo en el Bloque 34 del Parque Nacional Yasuní. Además, analiza las posibilidades que ofrecen alternativas como el Protocolo de Montreal para una más efectiva aplicación de medidas relativas a la preservación y conservación del medioambiente. Finalmente, se reflexiona sobre la responsabilidad de la comunidad internacional y el rol del Presidente de Ecuador, Rafael Correa, en el fracaso de la Iniciativa.

\section{- Palabras Clave}

Ecuador - Iniciativa Yasuní ITT- Cooperación medioambiental - Neoextractivismo - Petróleo

\section{Inglés | English}

Co-responsibility of the international community in the operation of Block 43 or ITT in the Yasuni National Park in Ecuador

\section{- Abstract}

This paper tackles the main difficulties that emerged as a result of the Yasuní ITT Initiative whereby Ecuador sought to avoid oil exploitation in the Block 43 of Yasunís National Park. Besides, it analyzes the possibilities that alternatives such as Montreal's Protocol offer for a more effective application of measures related to the preservation and conservation of the environment. Finally, it reflects on the responsibility of the international community and the role of the President of Ecuador, Rafael Correa, in the failure of the Initiative.

\section{- Key words}

Ecuador - Yasuní ITT Initiative - Environmental Cooperation - Neoextractivism - Oil

\footnotetext{
* Lic. en Relaciones Públicas y Comunicación Organización (Universidad Tecnológica Equinoccial, Quito, Ecuador). Maestranda en Integración y Cooperación Internacional (UNR, CEI/CERIR).
} 
"Bolsas de lágrimas secas arroyos contaminados las cosas son reales cuando se encuentran en sueños vemos sus armazones con la marca de Shell detrás de los escudos militares horcas perversas, horribles, llamadas torres de perforación petrolera que perforan nuestras almas. Pensamos que era petróleo, pero era sangre»

Nnimmo Bassey

\section{Introducción}

El Parque Nacional Yasuní ubicado en la llanura amazónica del Ecuador, posee una belleza inconmensurable: árboles, plantas, insectos, mariposas, invertebrados, vertebrados, ríos, comunidades indígenas con culturas ancestrales y "la sangre del diablo", petróleo. Considerando todos estos atributos, el entonces Ministro de Energía, Minas y Petróleo, Alberto Acosta, realizó una propuesta tan innovadora que no parecía provenir de este Ministerio: dejar el petróleo bajo tierra. Una Iniciativa concreta de esta índole generó un debate nacional e internacional de las repercusiones ambientales y económicas que este tipo de decisión representa para un país cuya economía depende en un alto porcentaje de la venta de este recurso. El presente trabajo, en primer lugar, realiza un acercamiento a la economía extractiva del Ecuador para luego poder entender la Iniciativa ITT y la importancia de su puesta en práctica.

Este trabajo está dividido en seis secciones: de manera inicial, se abordarán brevemente los "Antecedentes" para dar cuenta de la centralidad del extractivismo como sostén de la economía ecuatoriana y los intentos, por parte de la política nacional, de promover su diversificación. Posteriormente, se examinará la “Iniciativa Yasuní ITT", de donde nació la propuesta y cuáles fueron los nuevos paradigmas que se plantearon para generar visibilidad internacional. Luego, se analizará cómo se podría pasar "Del Protocolo de Kyoto a la Iniciativa ITT", es decir, cómo involucrar a la comunidad internacional en la preservación del Parque Nacional Yasuní. Este objetivo se lograría a partir del acercamiento de las agendas medio ambientales y la promoción de los beneficios globales que acarrearía la protección en conjunto. A continuación, en la sección "Gobier- no Nacional vs. Iniciativa ITT, ¿existió corresponsabilidad internacional?", se profundizará el objeto de estudio de interés, o sea, la falta de convencimiento de impulsar este proyecto por parte del Presidente Rafael Correa. Cuando un mandatario no está convencido de su propuesta ¿es posible que otros gobernantes lo estén? Tras estos interrogantes se retomarán algunos debates y en el apartado "Reactivación de la Iniciativa ITT ¿es posible?”se cuestionará la viabilidad de proteger este lugar único en el mundo. Finalmente, se plantearán unas "Conclusiones" de los temas abordados y se argumentará por qué es deseable inmiscuir a la sociedad civil en debates medioambientales ya que de esta manera se podría ayudar significativamente a mantener íntegro no solo el Parque Nacional Yasuní, sino toda la Amazonía Latinoamericana.

\section{Antecedentes}

La República del Ecuador ha realizado diferentes transiciones económicas desde que experimentó un boom cauchero, bananero y cacaotero. Es así que en la década de los setenta se pasó a un modelo de producción basado en la extracción de petróleo de la Amazonía. Este extractivismo ha obligado al Ecuador a mantener un modelo económico dependiente de sus recursos naturales para poder desarrollar proyectos dentro del territorio nacional. Esta situación ha traído aparejadas consecuencias nocivas como la destrucción de la selva y ha provocadola desaparición de comunidades indígenas o pueblos no-contactados ${ }^{1}$. La construcción de proyectos conocidos cómo la vía Auca y Maxus ${ }^{2}$ ha sido el inicio de problemáticas relacionadas con la contaminación de agua, reducción de hábitats, enfermedades crónicas, sincretismo que provoca que los pueblos originarios dejen de tener sus rituales ancestrales y que estas poblaciones desaparezcan en su totalidad o sea negada su existencia de acuerdo a las necesidades del gobierno de turno(FINER ET AL, 2010).

A pesar de las promesas de mejorar la vida de estos sectores desconocidos para gran parte de la sociedad civil por medio de las remesas del petróleo, no ha habido acciones

1. Se conoce de la existencia de pueblos que se encuentran en las profundidades de la Amazonía sin haber tenido contacto alguno con el mundo exterior, entre estos grupos se destaca especialmente a los Tagaeri y Taromenane. De igual manera, por la falta de acercamiento a estas comunidades se las ha llegado a considerar una leyenda dentro de las comunidades Huaoranis, tan sólo por la presencia de matanzas tribales y apoderamiento de lanzas se ha dado a conocer su paso en estos sectores inhabitados (Cabodevilla, 2016)

2. La vía Auca y Maxus son carreteras que fueron construidas para el uso de petroleras (Texaco y Maxus) para poder ingresar a los bloques petroleros en su poder. Este camino provocó la llegada de más petroleras, de colonos al sector, la deforestación y agotamiento de la tierra, la movilización de poblaciones Huaoranis. Actualmente estas vías siguen en uso y son las principales carreteras por las cuales se da la caza ilegal de animales exóticos con el fin de venderlos (Matt et al, 2010) 
concretas para efectivizarlas y lo que se ha podido observar, en todo caso, es que la pobreza se ha profundizado. En contraposición,los trabajadores petroleros se han visto favorecidos con la construcción de campos con las comodidades esenciales para trabajar en los bloques petroleros en las zonas de la Amazonía. Tala de bosques, matanza o tráfico de especies endémicas, contaminación de todos los recursos que están cerca de estos sectores son una constante. Adicionalmente,los pobladores se han vuelto mendigos de las empresas en su propio territorio (VILLAVICENCIO, 2010)

Actualmente, este modelo económico basado en el extractivismo de un solo recurso se ha vuelto insostenible por las condiciones particulares del crudo $^{3}$ que queda bajo tierra y por la caída en los precios de los hidrocarburos. En consecuencia, comienza a expandirse la idea de invertir en la creación de nuevas energías que tienen como beneficio ser renovables y a su vez, protegen el medio ambiente, teniendo en consideración que el petróleo es un bien que en pocos años estará en desuso o explotado en su totalidad. Es así que en 2007, la Presidencia del Ecuador dio a conocer una iniciativa innovadora y que llamó la atención de toda la comunidad internacional: dejar el petróleo bajo tierra. Esta declaración provocó diferentes reacciones y fue el primer paso para que se abra el debate de cuáles pueden ser las nuevas estrategias para reducir los gases de efecto invernadero (GEI) y otorgar a los países desarrollados su cuota de corresponsabilidad en la degradación del medio ambiente. La Iniciativa ITT ${ }^{4}$ buscaba frenar la extracción petrolera en los bloques Ishpingo, Tambacocha y Tipituni (por ello sus siglas) que se encuentran dentro del Parque Nacional Yasuní con el fin de mantener la biodiversidad de la zona, proteger a los pueblos no-contactados y así evitar que se propaguen los daños irremediables que la explotación de petróleo ha provocado en otros bloques concesionados y en funcionamiento.
Adicionalmente, la Iniciativa ITT buscaba compensar la "deuda ecológica" ${ }^{5}$ que tiene el mundo hacia el medio ambiente. A tal efecto, se realizaron los estudios pertinentes que arrojaron un valor monetario estimado que representaría el beneficio mundial de decidir preservar el crudo pesado en el subsuelo del Parque Nacional Yasuní. De todas maneras, está claro que ningún monto de dinero puede compensar el verdadero valor de la riqueza natural que posee el Yasuní. Paralelamente, con esta Iniciativa se buscaba cambiar el pensamiento clásico de los países cuyas economías están totalmente ligadas a la explotación de sus recursos naturales. Se intentaba reproducir esta idea en varios países en vías de desarrollo no sólo por la compensación monetaria, sino por replicar un modelo de respeto y adjudicación de derechos a la Naturaleza ${ }^{6}$.

\section{Iniciativa Yasuní ITT}

Alberto Acosta (ex Ministro de Energía, Minas y Petróleo) afirmaba que la Iniciativa Yasuní ITT era una idea construida colectivamente a partir de la intención del ex Presidente Lucio Gutiérrez de explotar los bloques petroleros que se encuentran dentro del Parque Nacional Yasuní. Por lo tanto, se podría considerar que desde el 2004 comenzó a crecer la idea de dejar el petróleo bajo tierra. Es así que en 2007, al poco tiempo de iniciar el periodo presidencial de Rafael Correa, el ex Ministro Alberto Acosta comenzó a desarrollar y moldear la idea de mantener el petróleo en el subsuelo de la Amazonía ecuatoriana y preservar 982 mil hectáreas de bosque húmedo tropical. De la mano de esta innovadora propuesta, en 2008 fue diseñada y reconocida una nueva Constitución Nacional que otorga derechos a la Naturaleza e impulsa la teoría del Sumak Kawsay (Buen Vivir). Entre sus artículos encontramos los siguientes derechos:

3. El petróleo que queda en el Yasuní es un crudo pesado de 14 API (American PetroleumInstitute). Este tipo de petróleo es más difícil de explotar debido a que se requiere más agua para sacarlo, ya que el mismo flota en ella. Es así que aun que se explote este crudo, lo esencial sería tener una refinería que permita utilizar este compuesto en la fabricación de derivados. Este tipo de crudo conlleva más pérdidas de ganancias a los países que no lo pueden refinar por su cuenta (Acosta, 2010)

4. La Iniciativa ITT se dio a conocer mediante un discurso del Presidente Rafael Correa ante la ONU el 25 de Septiembre de 2007.

5. La Deuda Ecológica es un profundo debate que no será ampliado en este trabajo debido a que no es el fin de este estudio. Más, se puede decir que la deuda ecológica es la responsabilidad que tienen los países industrializados por la destrucción paulatina del planeta como efecto de sus formas de producción y consumo, características del modelo de desarrollo, fortalecido con la globalización y que amenaza a la soberanía de los pueblos (Acción Ecológica, S/F)

6. Los derechos de la Naturaleza son otro debate que no será profundizado por no ser el fin principal de este trabajo. Se podría ampliar que los derechos de la Naturaleza marca un hito en la Constitución de 2008 en el Ecuador, poniendo atención en el problema ambiental ya generado y en el establecimiento de mínimas normativas para prevenir peores daños (Ávila Santamaría, 2010) 
Art. 71 "La naturaleza o Pacha Mama, donde se reproduce y realiza la vida, tiene derecho a que se respete integralmente su existencia y el mantenimiento y regeneración de sus ciclos vitales, estructura y funciones y procesos evolutivos. Toda persona, comunidad, pueblo o nacionalidad podrá exigir a la autoridad pública el cumplimiento de los derechos de la naturaleza. [...]"Constitución de Ecuador, 2008

\begin{abstract}
Art. 14 "Se reconoce el derecho de la población a vivir en un ambiente sano y ecológicamente equilibrado, que garantice la sostenibilidad y el buen vivir, Sumak Kawsay. Se declara de interés público la preservación del ambiente, la conservación de los ecosistemas, la biodiversidad y la integridad del patrimonio genético del país, la preservación del daño ambiental y la recuperación de los espacios naturales degradados." Constitución de Ecuador, 2008.
\end{abstract}

Una Constitución diferente, en la que por primera vez desde que Ecuador se conformó como República se interpreta a la Naturaleza como poseedora de derechos. Esta declaratoria sería lo que apuntale la propuesta de evitar la explotación de los recursos petroleros de la Reserva de la Biósfera ${ }^{7}$ Yasuní. Mediante la aprobación de la Constitución, el pueblo ecuatoriano demostraba su alta consciencia social y ambiental, lo que empoderaba las declaraciones que había realizado el 25 de Septiembre de 2007 Rafael Correa en el Foro de Presidentes sobre el Cambio Climático de las Naciones Unidas por medio del cual expresaba que "Ecuador ha traído una propuesta concreta e innovadora para contribuir a la reducción de emisiones de $\mathrm{CO} 2$ y a la conservación de la biodiversidad con nuestro proyecto Yasuní ITT.'

La Iniciativa Yasuní ITT, buscaba crear consciencia ambiental internacional sobre los efectos que tiene la explotación petrolera en el medio ambiente y otorgarles a los países desarrollados su cuota de corresponsabilidad por la emisión de gases de efecto invernadero en la atmósfera. El trabajo internacional que se realizó comenzó con un estudio de la cantidad de petróleo que se encontraba en el Parque Nacional Yasuní para poder determinar las estrategias que lograrían "vender" esta idea. Los estudios determinaron que existen 846 millones $^{8}$ de barriles de petróleo que representaban aproximadamente 410 millones de toneladas de $\mathrm{CO} 2$ emitidas a la atmósfera, por lo tanto, el costo total de la no-explotación del ITT rodeaba los 7.000 millones de dólares (LARREA, 2010). Se planteaba que la comunidad internacional financie el $50 \%$ del costo que significaría al Ecuador la no-explotación de ITT, lo que representaba un monto total de 3.500 millones de dólares a ser recaudados ${ }^{9}$ en trece años, tiempo que se estima podría durar la explotación de la reserva.

Adicionalmente, el proyecto tomó impulso en 2009 al tomar como ejemplo la idea de mantener el petróleo bajo tierra que se llevaba a cabo en Nigeria en donde 117 organizaciones se manifestaron ante el gobierno con la negativa de explotar cualquier nuevo pozo petrolero que se encuentre en sus tierras no solo por la degradación ambiental irreversible, sino también por las guerras civiles que se producen a causa de la corrupción y pobreza que provoca esta actividad (WRM, 2009). En efecto, si el dejar el petróleo bajo tierra se convertía en la lucha de varios pueblos, la Iniciativa ITT tomaba impulso y sería el postulado de acción global.

\section{Del Protocolo de Kyoto a la Iniciativa ITT}

Como se ha mencionado anteriormente, varias economías, especialmente en América Latina, África y Medio Oriente tienen como base principal de su modelo de producción la extracción de sus recursos naturales. Esta situación ha provocado que sus industrias no diversifiquen su giro de negocio y que la economía se torne dependiente de la fluctuación de los precios de estos bienes. Adicionalmente, las emisiones de las grandes industrias de los países desarrollados conjuntamente con los procesos que conllevan la perforación y extracción de estos recursos en los países de Tercer Mundo, han provocado mayor cantidad de gases de efecto invernadero. Como afirman Mendoza y Pérez (2010), "Cuando las fuentes se explotan de manera no sustentable provocan enormes daños a la sociedad y al medio ambiente, generando costos externali-

7. La UNESCO es el órgano que determina si un lugar puede ser denominado como Reserva de la Biósfera por sus características únicas. "Biosphere reserves are areas comprising terrestrial, marine and coastal ecosystems. Each reserve promotes solutions reconciling the conservation of biodiversity with its sustainable use." (UNESCO, S/F)

8. Al 14 de julio de 2016 mientras se escribía este trabajo, se dio a conocer que las reservas de petróleo en el Yasuní no son de 846 millones de barriles como se estimaba, actualmente se certifica que consta de 1.670 millones de barriles de petróleo. Información adquirida de la cuenta oficial de Petroamazonas en Twitter @PetroamazonasEP el día en mención

9. Se pensaba adquirir apoyo monetario mediante aportes tanto públicos como privados en estancias nacionales e internacionales. Dicho dinero se pondría en un Fideicomiso que regule la ONU para proyectos en el territorio nacional. 
zados que por lo general no son considerados en el precio final de la energía generada." El aprovechamiento de recursos naturales no sólo provoca su agotamiento, sino que también conlleva efectos colaterales como la pérdida de bosques, extinción de especies de animales, contaminación de fuentes de agua y desaparición de comunidades indígenas. Los países que son primordialmente extractivistas no toman en cuenta todas las variables que engloba la explotación de estas riquezas naturales debido a lo fácil que se ha vuelto perforar un nuevo pozo petrolero. En efecto, no se busca invertir en nueva tecnología que a su vez sea renovable debido a que no tienen una visión a largo plazo de lo que este esquema podría significar para el medio ambiente y su propia economía.

Cabe destacar que varias convenciones y protocolos internacionales se han desarrollado para poner en la palestra pública la problemática ambiental que se ha estado viviendo a partir de la Revolución Industrial. De hecho, en 1987 se firmó el Protocolo de Montreal para encontrar formas de mitigar la emisión de clorofluorocarbono que comenzaba a crear un agujero en la capa de ozono. Este Protocolo tuvo un reconocido éxito en la disminución y prohibición en el uso de agentes que dañaran la atmósfera debido a las restricciones comerciales y limitaciones temporales que se manejaron para su cumplimiento. Según Barrett "normalmente, las sanciones comerciales perjudican al país que impone, una razón por la que con frecuencia son ineficaces. Pero la amenaza de Montreal parece dar resultado ¿por qué? Porque si las sanciones disuaden la reubicación de la producción o las emisiones, entonces los países que imponen las sanciones ganan al imponerlas. Esto, a su vez, refuerza la credibilidad de las sanciones." (2000). El Protocolo de Montreal ${ }^{10}$ se podría considerar como la propuesta básica para el diseño de nuevas convenciones de cambio climático, reducción de emisiones y sanciones. Pero, conforme han pasado los años, los acuerdos han sido más permisivos y no tienen cláusulas claras con limitaciones definidas.
Después de Montreal, se suscitaron nuevas preocupaciones referentes al cambio climático. En 1997, durante la Conferencia Marco de Cambio Climático de Naciones Unidas (CMNUCC)se diseñó el Protocolo de Kyoto, por medio del cual se buscaba la reducción del $5 \%$ de gases de efecto invernadero entre los años 2008 y 2012.Pero al no considerar cláusulas que definan limitaciones comerciales como lo estipula el Protocolo de Montreal, se constató que en lugar de una reducción de las emisiones, éstas aumentaron un 16\% entre 1997 y 2004. Es así que en 2005 al no reducirse las emisiones de GEI y al tener el compromiso de varias naciones que englobaban el 55\% de emisiones, Kyoto entró en vigor ${ }^{11}$. Lastimosamente dentro de la ratificación del compromiso de disminución del uso de agentes que provoquen GEI países como China y Estados Unidos que son considerados los principales contaminantes del mundo no han tomado su cuota de corresponsabilidad para poner en práctica este mandato (HUMPHREY, 2009)

Dentro de las afectaciones que produce el dióxido de carbono en la atmósfera se destaca de manera particular el calentamiento global. Esta problemática no afecta expresamente al país que lo genera, de hecho, "el dióxido de carbono, el gas principal causante del cambio climático global. Es muy estable y permanece en la atmósfera durante unos 60 años después de su emisión. Se mezcla con facilidad y a los pocos meses el dióxido de carbono que fue emitido en Nueva York o Beijing estará esparcido alrededor del mundo." (HEAL, 2000). El dióxido de carbono se ha convertido en un bien público mundial ${ }^{12} \mathrm{y}$, por lo tanto, debería llevar a los países a trabajar arduamente para reducir las emisiones contaminantes y evitar los daños colaterales de estos procesos. De igual manera, un protocolo más estricto como lo fue el de Montreal sería esencial para que los países desarrollados tomen su cuota de corresponsabilidad en esta problemática.

Se puede mencionar el Artículo 2 Inciso IV del Protocolo de Kyoto en donde se destaca la "investigación, pro-

10. Si bien las sanciones comerciales que se manejan en el Protocolo de Montreal son más morales que monetarias, sus restricciones para importación y exportación de agentes que dañen la capa de ozono ha tenido un reconocido éxito. Lastimosamente, mientras haya demanda de estos productos, se los podrá encontrar en el mercado o se dará el tráfico de los mismos.

11. Para que el acuerdo entrase en vigor, ha sido necesario que 55 naciones que representan el $55 \%$ del total mundial de las emisiones de gases de efecto invernadero lo hayan ratificado. En la actualidad 164 países lo han ratificado o aceptado, lo que supone más del 61\% de las emisiones, según datos de la UNFCCC. El acuerdo entró en vigor el 16 de febrero de 2005, después de la ratificación por parte de Rusia el 18 de noviembre de 2004 (UNFCCC, S/F)

12. Una característica de los bienes públicos (mundiales o no) es la no-rivalidad: el consumo del bien por una persona no reduce su disponibilidad para el consumo de otras ni supone un coste adicional. Otra característica es la no-exclusividad: es imposible impedir a alguien el consumo de ese bien. Los bienes públicos mundiales se pueden dividir en recursos naturales (océanos, atmósfera, espacio exterior), sociales o artificiales (ciencia, cultura, tradiciones, Internet), y "políticos" (paz, salud, estabilidad financiera) (World Governance, S/F) 
moción, desarrollo y aumento del uso de formas nuevas y renovables de energía, de tecnologías de secuestro del dióxido de carbono y de tecnologías avanzadas y novedosas que sean ecológicamente racionales"(1998) Este apartado fue uno de los pilares para la Iniciativa ITT, ya que se buscaba el cambio de matriz productiva para desarrollar nuevas fuentes de energía que a su vez sean amigables con el medio ambiente.

Adicionalmente como lo manifiesta Barrett, "el protocolo de Kyoto permite a un país signatario en el Anexo I cumplir su límite de emisión al pagar a un país en vías de desarrollo para que lleve a cabo una reducción progresiva en su nombre"(2000). Con este enunciado podemos apreciar que Ecuador al ser un país en vías de desarrollo podría beneficiarse del pago de un país desarrollado para reforestar un área específica y a su vez explotar sus recursos naturales mientras exista una reducción de GEI. Sin embargo, la Iniciativa ITT iba más allá proponiendo al mundo no explotar sus reservas de petróleo si la comunidad internacional apoyaba con el 50\% que significaba la explotación de estos recursos. En efecto, la Iniciativa ITT buscaría la financiación internacional mediante un fideicomiso para la conservación de un Parque Nacional y paralelamente el país se mostraba ambientalmente consciente de su corresponsabilidad al financiar el otro 50\% del proyecto.

Al publicitarse la Iniciativa ITT como un proyecto que buscaba cambiar la matriz productiva de un país mediante la conservación del ecosistema, se podría pensar que el Ecuador estaba totalmente consciente de las implicaciones que tiene el dióxido de carbono en la atmósfera y como éste en varios años se ha transformado en un bien público mundial que debe ser tenido en cuenta para la supervivencia del planeta. Heal manifiesta que "en el caso de bienes públicos mundiales cuya provisión requiere nuevas normas técnicas, lograr que uno o dos países grandes sean los primeros en adoptarlas facilita mucho la amplia adopción de las nuevas normas apropiadas" (2000). En efecto, es el apoyo de potencias mundiales lo que lleva a que proyectos, protocolos o tratados sean firmados para promover acciones que colaboren en resolver diferentes problemáticas mundiales en ámbitos como por ejemplo, salud, educación, terrorismo, medio ambiente. Por este motivo, Ecuador centró su plan de acción, en primera instancia en la Unión Europa, para luego difundirla en Estados Unidos y finalmente en Asia.

Cabe destacar que la comunidad internacional posee la capacidad de enfrentar altos costos para el tratamiento de algunas cuestiones como el terrorismo (en cuanto a defensa, armas, movilización de tropas, etc.) pero no se ha logrado tomar plena consciencia del alto costo que el caos medio ambiental provoca globalmente. Kelly Swing, científico de la Reserva Tiputini ${ }^{13}$ en entrevista con el diario The Guardian manifestaría "dado que el mundo gasta 3 billones de dólares en tabaco cada dos días, algunos billones de dólares para salvar millones de especies podría haber sido la mejor apuesta en la historia de la conservación" (2013) Las prioridades del mundo se ven reflejadas en sus gastos, es por ello que el cambio climático ha pasado a un segundo plano y tan solo cuando los problemas parecen ser irreversibles es cuando se toman acciones. El mayor ejemplo que se posee sobre la eficacia de cláusulas claras y limitaciones comerciales es Montreal, ya que gracias a su supervisión constante se ha podido observar, treinta años después, una reducción en el agujero de la capa de ozono, unos 4 millones de kilómetros de su diámetro (SOLOMON ET AL, 2016)

Las acciones conjuntas que realiza la comunidad internacional para mitigar las problemáticas globales son esenciales para tener beneficios. Susan Solomon del Massachusetts Institute of Technology (MIT) en relación con la disminución del agujero de la capa de ozono declaró "Es una magnífica noticia para nosotros, ¿no? ¿No somos asombrosos los humanos?, que hacemos algo que crea una situación concreta y decidimos colectivamente, como mundo, ¡vamos a acabar con esas moléculas! Y lo hacemos, y ahora vemos que el planeta está respondiendo" (2016). Éste es un claro ejemplo de cómo los actores internacionales pueden unirse para reducir las emisiones de gases que dañan la atmósfera o también determinar cómo sanciones reales podrían ser totalmente eficientes. Sería fundamental replicar en el Protocolo de Kyoto el ejemplo de Montreal para disminuir los GEI y así reducir el calentamiento global. Pero, estos cambios no vendrán si no existen verdaderas restricciones para los países contaminantes ${ }^{14}$, o si estas naciones siguen transfiriendo su cuota de responsabilidad a pequeños territorios.

13. Reserva diseñada por la Universidad San Francisco de Quito dentro del Parque Nacional Yasuní con el fin de estudiar e investigar este lugar único en el mundo. Así han llegado a entender a profundidad la naturaleza, logrando diseñar planes de preservación(USFQ, 1994)

14. El 30 de Noviembre de 2016 los países que conforman la OPEP decidieron reducir la producción de petróleo debido a la caída del precio de éste, no por razones medioambientales. Es así que se afirma que la reducción en la producción beneficia a todos los países que producen e importan petróleo al obtener un precio alto y estable para este recurso. (Biroh, 2016) Con ello se puede afirmar que tan sólo se disminuye la explotación petrolera cuando el precio no ayuda a éste negocio más no por motivos ligados al cambio climático. 
En 2009, el gobierno del Ecuador y el Parlamento Alemán ${ }^{15}$ conjuntamente con las Naciones Unidas y el Programa para el Desarrollo (PNUD) diseñaron el documento "Yasuní ITT: Una Iniciativa para Cambiar la Historia" en el cual se da a conocer la propuesta de la Iniciativa de la siguiente manera:

"Esta original iniciativa plantea:

a. Una opción innovadora para combatir el calentamiento global, evitando la explotación de combustibles fósiles en áreas de alta sensibilidad biológica y cultural en los países en desarrollo;

b. La protección de la biodiversidad en Ecuador y el apoyo al aislamiento voluntario de las culturas indígenas no contactadas que habitan el Parque Yasuní (TAGAERI y TAROMENANE);

c. El desarrollo social, la conservación de la naturaleza y la implementación de fuentes renovables de energía, en una estrategia encaminada a consolidar un nuevo modelo de desarrollo equitativo y sustentable en el país." ${ }^{16}$

Al tener la propuesta formulada y las estrategias marcadas, el grupo negociador base ${ }^{17}$ podía delimitar su trabajo y hacer que la Iniciativa ITT tomara fuerza. De igual manera, teniendo como referente al Protocolo de Kyoto y a su vez los Objetivos de Desarrollo del Milenio (ODM) ${ }^{18}$ propuestos por ONU, lo que se esperaba era una respuesta positiva que colectivamente le diera a este documento un mayor impulso para promover la Iniciativa ITT. En efecto, el lograr la vinculación de los ODM con el Yasuní, era una manera de concatenarse con el cumplimiento del Séptimo Objetivo: Garantizar la sostenibilidad del medio ambiente (ONU, 2000).

En forma simultánea, se pensaba que al haber proclamado la UNESCO al Parque Nacional Yasuní como Reserva de la Biósfera la preservación de este lugar iba a ser más importante y tendría una participación más activa de la Organización de Naciones Unidas para evitar la explotación de este lugar. Efectivamente, se preveía que al tener una propuesta acorde con los propósitos de la Carta de Naciones Unidas, del Protocolo de Kyoto y los ODM, estos estamentos internacionales se transformarían en un aliado para la Iniciativa. Por otra parte, se consideraba que el título de Reserva de la Biósfera no sólo serviría de documento decorativo en el Ecuador.

Lastimosamente existen personas dentro de estos organismos que no consideran importante impulsar estas propuestas de manera firme o intervenir con mayor fuerza en las problemáticas de un país. Así se puede mencionar a Gabriel Jaramillo (responsable del Área de Ambiente y Energía - PNUD en Ecuador) quien manifestó que "la ONU no es la policía del mundo". ${ }^{19}$ Esta cuestión lleva a pensar sobre el real compromiso que muestra el personal que se encuentra dentro de la institución. Si bien no se puede afirmar que todo el organismo piense de esta manera, a partir de este tipo de declaraciones se puede interpretar que la Iniciativa ITT no representaba un beneficio para la conservación del medio ambiente.

Como se mencionaba anteriormente, el $\mathrm{CO} 2$ se ha convertido en un bien público mundial cuando se lo debería catalogar como mal público mundial. Como han manifestado Jayaraman\&Kanbur, "consideremos un tipo de bienes públicos que parece satisfacer la caracterización de tecnología aditiva: aire limpio, o su opuesto, el mal público, los gases de invernadero. Aquí, quienquiera que reduzca sus emisiones, beneficia a todo el mundo" (2000). La necesidad de adherirse a la Iniciativa ITT no solo representaba la no-emisión de 410 toneladas de dióxido de carbono en la atmósfera, de la mano, se lograba conservar el ecosistema único de este lugar y proteger a los pueblos no-contactados.

Si bien el Protocolo de Kyoto ha servido de marco para poder potenciar la necesidad de crear un ambiente sano y sustentable para la humanidad, la falta de sanciones reales ha hecho que los países tomen con ligereza las acciones a implementar y descarten propuestas alternativas. Se debería replicar el modelo del Protocolo de Montreal para avanzar hacia una sociedad internacional que tome con seriedad su cuota de corresponsabilidad en la afectación del medioambiente, y no deje de lado las nuevas posibilidades de dejar el petróleo bajo tierra.

15. El Parlamento Alemán fue el primero en discutir su adhesión a la Iniciativa ITT, es así que se comprometió a entregar 50 millones de euros por año durante los trece años en que el petróleo podría ser explotado. (Boaventura de Souza, 2009)

16. Se respeta la negrilla como marca el texto original.

17. El tiempo que duró la Iniciativa ITT, ésta contó con 5 grupos negociadores con diferentes actores. Es así que Roque Sevilla sería la primera persona en encargarse de la ardua tarea de formular las acciones a realizar.

18. Compromiso de 191 Jefes de Estado en donde se fijaron ocho objetivos que son las metas cuantificadas y cronológicas, que se han fijado para luchar contra las problemáticas actuales (ONU, 2000)

19. Entrevista realizada por la autora a Gabriel Jaramillo en la Ciudad de Quito el 20 de junio de 2016. 
Lastimosamente, a pesar de que la Iniciativa ITT respondía a varios de los postulados internacionales para la conservación del medio ambiente mediante proyectos autosustentables ésta llegó a su fin en 2013 por la falta de vinculación internacional y porque no se llegó a recaudar el dinero deseado. En ese momento los organismos internacionales y la Unión Europea fueron considerados como los causantes del fin de la Iniciativa ${ }^{20}$. En este contexto se ha dado paso a nuevas discusiones y mayores interrogantes del costo-beneficio ${ }^{21}$ de la extracción de los recursos naturales.

\section{Gobierno Nacional vs. Iniciativa ITT ¿exis- te corresponsabilidad internacional?}

El 15 de Agosto de 2013 llega a su fin la Iniciativa ITT con las tan recordadas palabras del presidente Correa: "la comunidad internacional nos ha fallado"22 ¿¿Cómo se llegó a este momento? Para encontrar parte de la explicación habría que recordar todas las "Sabatinas" ${ }^{23}$ en las que un día el Presidente estaba a favor de la Iniciativa y al siguiente la menospreciaba. De igual manera, sería necesario hacer memoria de las veces que mandaba a la comunidad internacional a "meterse sus moneditas en las orejas", declaraciones impropias para la seriedad con la que debía presentarse la Iniciativa ITT. Evidentemente, si el Primer Mandatario no demostraba el verdadero valor que representaba conservar el Parque Nacional Yasuní, no sólo para el Ecuador sino para el mundo entero, la respuesta internacional no podía ser favorable, a pesar de haber recibido un gran apoyo en su inicio. Sin un convencimiento sincero por parte de la Presidencia de Ecuador iba a resultar muy difícil convencer a potencias mundiales de la necesidad de sentirse responsables de preservar este lugar.

Los arrebatos y doble discurso del Presidente llevaron a la comunidad internacional a dudar de la Iniciativa ITT y además provocaron la salida del grupo negociador base, lo que, a su vez, creó desconfianza en la comunidad internacional. Tal vez este cambio constante de personal fue una de las mayores limitaciones para la cooperación internacional en la Iniciativa ITT ya que se abandonó el trabajo metódico que llevaba el grupo negociador base y se comenzó a llevar el proyecto como "pura farándula"24. Como lo manifestó Malki Sáenz, integrante del grupo negociador base:

La cara visible es muy importante (gente respetada y respetable) esta es una iniciativa de confianza [...] delineaste una estrategia, donde ir, cuando ir, cuanto pedir, donde trabajar, trabajar en las esferas altas de los acuerdos binacionales, trabajar en la sociedad civil de esos países, tener encuentros masivos con los embajadores para que sepan de que se trata la iniciativa, hacer que cancillería se convierta en una diplomacia verde que pueda vender bien el producto [...] pasar de eso a un tema de farándula probablemente tengas más repercusión mediática pero en sí fondos que lleguen al fideicomiso no iban a llegar. ${ }^{25}$

En 2011 se creó el Manual de Procedimientos para el Funcionamiento del fideicomiso ${ }^{26}$ que sería el respaldo que utilizaría el Ecuador para demostrar la seriedad de la Iniciativa ITT. De la mano del PNUD, se presentaron los planes a desarrollarse con el dinero que se recibiría de la esfera internacional. De hecho, se establecía que "permitirá que el Gobierno enfrente los retos del cambio climático y del desarrollo sustentable al cambiar la matriz energética del país, a través de inversión en proyectos energéticos renovables inclusivos ambientalmente amigable tales como plantas de energías hidroeléctricas, geotérmicas, solares, eólicas, de biomasa y de mareas" (MANUAL DE PROCEDIMIENTOS, 2011: Pp.2-5) Mediante estos objetivos planteados se podía demostrar que los fondos recaudados irían a fomentar cambios estructurales en la industria ecuatoriana y mejorar las condiciones de vida de los ciudadanos, logrando así el proclamado "Buen Vivir". Lastimosamente, al tratar esta Iniciativa como una simple recaudación de fondos se generó que el inicial entusiasmo que demostró la comunidad internacional se vaya enfriando y dejó en evidencia la carencia de compromiso del Gobierno y la falta de una estrategia a largo plazo del nuevo grupo negociador.

Paralelamente a las donaciones que llegaban al fideicomiso se podían comprar Certificados de Garantía Yasuní (CGY) los cuales servirían a las naciones para demostrar

22. Cadena de radio y televisión realizada por la Presidencia de la República del Ecuador el 15 de Agosto de 2013.

23. Intervenciones que hace el presidente ecuatoriano todos los sábados en cadena nacional.

24. En 2010 Ivonne Baki asume como Jefe Negociador en la Iniciativa ITT, varios aseguran que ella fue quien dejó morir el proyecto debido a su falta de acercamiento con el trabajo que se llevaba realizando y tan sólo se centró en dar a conocer la idea con sus allegados y amigos diplomáticos. 25. Entrevista realizada por la autora a Malki Saénz en la Ciudad de Quito el 21 de Junio de 2016

26. El dinero sería depositado en un fondo fiduciario establecido bajo el techo de ONU para la preservación de áreas protegidas, programas de reforestación, la expansión de energías renovables, programas de ahorro de energía y proyectos sociales. (Dilger, 2010) Mas el problema se suscitó cuando el Presidente Correa mencionó que la ONU pretendía imponerle en que utilizar estos fondos. 
su compromiso con el Protocolo de Kyoto en la adquisición de cuotas de emisiones de gas de carbono. Este certificado también era un comprobante fehaciente de que en el caso de ser explotado el Yasuní se reembolsaría la inversión realizada. Como expresen Acosta et al., "en el caso de que en el futuro algún gobierno ecuatoriano extrajera el crudo, este petróleo debería ser comercializado para financiar los aportes recibidos." (2010, Pp.123) El fideicomiso y los CGY fueran las garantías con las que se buscó dar confianza a la comunidad internacional de que su dinero sería bien utilizado. Sin embargo, al dejar de establecer las conversaciones frecuentes y al no continuar el contacto con los inversionistas, la Iniciativa ITT perdió fuerza y credibilidad en la esfera internacional.

Al llegar el 2013 se habían recaudado 13.3 millones de dólares y un compromiso de 116 millones de dólares, y, personas cercanas al gobierno acusaron a la crisis monetaria mundial como el factor clave para que no haya llegado el dinero comprometido. Roque Sevilla, quién fue el primer responsable de la Iniciativa, afirmó que las contradicciones del Gobierno y el mantener siempre bajo la manga el Plan $\mathrm{B}^{27}$, es lo que desanimó a los accionistas, el chantaje ${ }^{28}$ no podía ser el punto clave para mantener el apoyo internacional (2010). Al contrario provoca que se den declaraciones como las que realizaría Sebastián Lesch, portavoz del Ministerio de Cooperación alemán a DW: "nuestra pregunta principal es si esto estaría sentando un precedente para otros gobiernos, nuestro objetivo es implantar políticas activas para países activos, no pagarles para que no hagan nada"29(2010). Algunos estamentos internacionales llegaron a considerar que el Gobierno del Ecuador sólo buscaba recaudar dinero sin realizar verdaderas acciones que conlleven al cambio de matriz productiva. La Iniciativa ITT estaba bajo la lupa no sólo nacional, sino internacional y su fracaso significaría que otros países como Perú, Bolivia, Venezuela, Guatemala o Nigeria, no puedan replicar esta idea y sean afectadas por una desacertada campaña que llevaba a pensar que el dejar el petróleo bajo tierra era un negociado de los países en vías de desarrollo.

Entre altos y bajos la Iniciativa ITT llegó a su fin, lo que provocó que varios colectivos ambientalistas y grupos de la sociedad civil busquen maneras de frenar la explotación de este lugar. Por un lado se aseguraba que, "en el caso Yasuní, el gobierno ha presentado dos mapas. En uno, anterior a la propuesta gubernamental de explotar el Yasuní, los pueblos no contactados están en el Parque Nacional Yasuní. Seguramente este mapa servía de argumentos para pedir la ayuda internacional. El otro mapa, posterior al decreto presidencial que anuncia la explotación, los pueblos no contactados están fuera del límite imaginario." (ÁVILA SANTAMARÍA, 2015) Adicionalmente se apelaba al Artículo 407 de la Constitución que manifiesta:

\begin{abstract}
"Se prohíbe la actividad extractiva de recursos no renovables en las áreas protegidas y en zonas declaradas como intangibles, incluida la explotación forestal. Excepcionalmente dichos recursos se podrán explotar a petición fundamentada de la Presidencia de la República y previa declaratoria de interés nacional por parte de la Asamblea Nacional, que, de estimarlo conveniente podrá convocar a consulta popular"30
\end{abstract}

Con este Artículo se buscó recolectar firmas para lograr el llamado a referéndum que permita a la sociedad civil determinar si era necesario explotar el Parque Nacional Yasuní. Se buscó de otras maneras llegar a las Conferencias sobre Cambio Climático para dar a conocer la necesidad de preservar este lugar para mitigar el cambio climático. Más se pudo observar que se manipularon mapas, información y artículos constitucionales para sepultar todas las propuestas y todo el imaginario que se creó alrededor de la Iniciativa ITT.

Es así que Correa envió a la Asamblea Nacional un escrito en el cual declara de "Interés Nacional" la explotación del bloque ITT. Diferentes actores sociales podrían considerar que las decisiones tomadas el 03 de octubre de 2013 por parte de la Asamblea Nacional de aprobar la "Declaratoria de Interés Nacional para la explotación petrolera de los Bloques 31 y 43 situados dentro del Parque Nacional Yasuní" fueron apresuradas y no tomaron en consideración la expectativa nacional de poder llegar a un referéndum que permita al pueblo decidir si realmente existía ese interés. En efecto, haber dado por terminada la Iniciativa ITT en el mes de Agosto de 2013 y declararla de interés en Octubre del mismo año fue lo que llamó la atención de los colectivos sociales que venían trabajando arduamente en esos dos meses para buscar el referéndum nacional que no se llevó a cabo.

27. Roque Sevilla ha dado a conocer en varias entrevistas y artículos que Correa mantenía como Plan A la recaudación de fondos para preservar el Yasuní. Y en su defecto el Plan B era la explotación de estos bloques petroleros.

28. Roque Sevilla de igual manera afirma que Correa manejaba un chantaje con la comunidad internacional en donde si le entregaban fondos no explotaba el Yasuní caso contrario lo explotaba.

29. Texto original de las declaraciones del portavoz del Ministerio de Cooperación alemán "One of our main questions is whether this would be setting a precedent for other governments," BMZ spokesman Sebastian Lesch told Deutsche Welle. "Our objective is to pursue active policies for active countries, not pay them to do nothing."(2010)

30. Constitución de Ecuador, 2008 
Podemos recordar que el Artículo 407 que demuestra el compromiso ambiental que se mantenía en el Ecuador fue lo que han reclamado constantemente los colectivos ambientalistas ${ }^{31}$ ya que no se les dio la posibilidad de recaudar firmas para lograr la Consulta Popular para proteger el Parque Nacional impidiendo su explotación. Como se ha mencionado anteriormente, la explotación de recursos naturales no solo provoca cambios en la biósfera, sino que a su vez, no ha demostrado ser eficiente para resolver problemas sociales de las zonas afectadas. Al contrario, se profundiza la pobreza de la zona y además se dan con más frecuencia enfermedades crónicas debido al uso de agentes químicos tóxicos provenientes de esta explotación provocando en muchos casos leucemia, tuberculosis, desnutrición, malformaciones congénitas, cáncer a la piel, estómago, testículos y pulmones (SOLIZ, 2015)

Simultáneamente la Asamblea Nacional intentó minimizar la afectación que sufrirá el Parque Nacional Yasuní al afirmar que no se afectaría al mismo en una extensión mayor al uno por ciento de la superficie actual pero como manifiesta Soliz, "además de constituir un insulto para la inteligencia del pueblo, la respuesta limita a la visión romántica de preservar el Yasuní desde su biodiversidad y belleza natural." (2015, Pp.78). El dar a conocer que sólo una mínima porción de terreno será afectada para la explotación del Yasuní limita la capacidad de racionalizar lo que realmente significa ese uno por ciento, es decir, deforestación, ríos contaminados, aniquilación de especies endémicas por la presencia humana, desaparición de insectos (ya que las antorchas los atraen y los matan), contaminación de terreno que provoca que no se pueda sembrar; movilización, contagio de enfermedades o matanza de pueblos indígenas propios de la zona.

Después de las innumerables actuaciones del Gobierno para desacreditar a movimientos sociales y ambientales sobre la necesidad de la no-explotación del Yasuní, se puede constatar que se dejaron de lado los postulados del Protocolo de Kyoto y los ODM que se buscaron en un principio para lograr la adhesión internacional. Si bien las Conferencias Marco de Cambio Climático fueron to- madas como pilar por manifestar la necesidad de crear fuentes renovables de energía, mantener los bosques y así evitar los gases de efecto invernadero, la Iniciativa ITT es un claro ejemplo de la falta de compromiso que presentó el Estado ecuatoriano para mantener sus propuestas. En la búsqueda de preservación de Parques Nacionales como el Yasuní, no se puede culpar a la comunidad internacional por la falta de agilidad que presentó el Gobierno Nacional al no darle el reconocimiento, impulso y tiempo que necesitaba para implementarse esta iniciativa ambiental.

El Gobierno Nacional insiste en la corresponsabilidad internacional en la explotación del Parque Nacional Yasuní para lo que se debería recordar el principio de la Convención Marco de las Naciones Unidas sobre el Cambio Climático de 1992 "responsabilidades compartidas pero diferenciadas". Es por ello que tratar de justificar la falta de agilidad y las claras intenciones ${ }^{32}$ de explotar estos bloques desde un inicio, es irracional, la responsabilidad de la explotación del bloque ITT es completamente del Gobierno Nacional al cual en palabras de Alberto Acos$\operatorname{ta}^{33}$ "le quedó grande la Iniciativa ITT". Este tipo de proyectos no se pueden desarrollar de la noche a la mañana o como esperaba Correa en los seis años (entre el 2007 y 2013) que estuvo vigente la Iniciativa. Como se mencionaba anteriormente la degradación de la atmósfera llevó años, su reparación llevará décadas como lo sucedido con el agujero de la capa de ozono. Adicionalmente, es el Gobierno Nacional el que no permitió que se lleve a cabo la Consulta Popular que promovían las organizaciones ambientales para definir el interés nacional de la explotación del Parque Nacional Yasuní. En efecto, organizaciones ambientales, movimientos sociales y personas entrevistadas como Alberto Acosta, Malki Sáenz, Carlos Larrea, Andrés Páez, consideran que de haberse dado la Consulta Popular la respuesta hubiera sido un rotundo $\mathrm{NO}$ a la extracción petrolera.

31. Acción Ecológica y Yasunidos fueron dos de los grupos ambientalistas clave en el momento en el cual se buscaba impedir la explotación del Yasuní mediante la recolección de firmas que llegaran a la Asamblea con el fin de llamar a referéndum y el país pueda decir si se explotaba o no estos bloques petroleros. Adicionalmente se daban protestas en la ciudad de Quito para poner en conocimiento de todos los habitantes de esta intención de sacar el petróleo del ITT. Es conocido que no se aceptaron las actas que se quería diseñar para la recolección de firmas y las pocas recaudadas para el pedido de referéndum fueron dadas de baja o negada su participación.

32. La explotación del Bloque ITT "va porque va", ya que siempre estuvo en la agenda de Correa. La propuesta de no explotar el crudo fue sólo una coartada distractora para entretener a algunos defensores de la biodiversidad. Ojalá, algún día, cuando la resistencia social sea más fuerte, la voz de la naturaleza se imponga para impedir la destrucción de una de las últimas fronteras verde-humanas del planeta, el Yasuní. Para entonces, espero que puede haber un "entonces". (Villavicencio, 2010)

33. Entrevista realizada por la autora a Alberto Acosta el 13 de Junio de 2016 en la Ciudad de Quito. 


\section{Reactivación de la Iniciativa ITT ¿es posible?}

"Yasuní significa Sagrado, Yasunizar es Volver a lo Sagrado"34

La Iniciativa ITT concluye como una amarga experiencia que exhibe cómo la ambición de grupos petroleros de los países desarrollados va a primar frente a la necesidad de replicar cambios en materia medioambiental. También ha demostrado que a base de limitaciones comerciales es cómo se obtienen respuestas y compromisos globales, que, muchas veces y más en temas que inciden en el medio ambiente, no tienen resultados inmediatos sino que responden a un largo proceso que suele tomar de dos a tres veces el tiempo que se demoró en producir.

Personalmente, se considera que una agenda de cooperación internacional para cuidar el medio ambiente es primordial para evitar más daños al ecosistema y la pérdida de biodiversidad y culturas únicas. Crear frentes binacionales o regionales sería una solución esencial para poder defender los bosques húmedo tropicales, preservar la biodiversidad y cuidar la vida de los pueblos no-contactados. Ya que si tomamos en consideración que "muchas cuestiones del control del medio ambiente atraviesan, inherentemente, fronteras nacionales" (COOK \& SACHS, 2000), se buscaría aunar esfuerzos de varios países limítrofes que posean características similares. En efecto, las similitudes de territorios contiguos pueden transformarse en el motivo principal para poder trabajar conjuntamente $\mathrm{y}$ crear proyectos que tengan como fin cambiar la situación de toda la región y el mundo.

Es necesario recordar que en 1978, se creó la Organización del Tratado de Cooperación Amazónica (OTCA), en el cual se manifiesta que "CONSCIENTES que tanto el desarrollo socio-económico como la preservación del medio ambiente son responsabilidades inherentes a la soberanía de cada Estado, y que la cooperación entre las Partes Contratantes servirá para facilitar el cumplimiento de estas responsabilidades, continuando y ampliando los esfuerzos conjuntos que están realizando en materia de conservación ecológica de la Amazonia" (TRATADO DE COOPERACIÓN AMAZÓNICA, 1978). Las naciones que participan en este tratado son Bolivia, Brasil, Colombia. Ecuador, Guyana, Perú, Suriname y Venezuela, países que comparten en pequeñas o grandes porciones las riquezas de este bosque húmedo tropical. Se insiste en el Protocolo de Montreal, si lo trasladamos a organismos y tratados regionales se podría lograr mejores agendas de cooperación sostenibles para mitigar el cambio climático.

Se considera que partir de un trabajo nacional bien desarrollado se podría pasar a uno binacional para después ir agregando a otros países participantes hasta transformar a la Iniciativa ITT en una Iniciativa Amazonas. Este apartado puede sonar bastante utópico, pero sería un primer paso que ayudaría a que las prioridades de todas las naciones que comparten este gran espacio de América del Sur se unan para trabajar en la preservación de este espacio que reúne muchísima flora, fauna y comunidades indígenas. Poder aunar esfuerzos e ideales podría ser la forma en la cual no se tenga que pensar en la forma de recuperar estos sectores. Si bien se puede pensar en la reforestación o el abandono de proyectos petroleros, los daños irreversibles que se mencionaron anteriormente solo se van a ahondar y no será recuperable todo lo que se ha perdido debido a la extinción de estos recursos. Es por esto que si países como los del OTCA lograran salvaguardar la Amazonía como un bien público mundial el planeta entero sería beneficiario de una reducción en el calentamiento global, se evitaría el uso inapropiado de estos sectores y se impediría el colapso ambiental.

El trabajo conjunto y consensuado tendría un alto impacto ya que éste llevaría a muchos beneficios a largo plazo, pero países como Estados Unidos y China no quieren unir esfuerzos porque otras naciones sin hacer nada serían también beneficiarios de este trabajo con el tan llamado "free-riders", es decir, todo lo que se haga a favor del medio ambiente ayudará a todo el mundo a ser partícipe de un mejor ecosistema (VOGLER, 2014). Lastimosamente muchos tratados y organismos internacionales no tienen fuerza o un liderazgo para superar las problemáticas compartidas. Como se ha anotado anteriormente, todos estas reuniones se han realizado para definir la agenda de trabajo para proyectos conjuntos, más de papel y objetivos escritos no se ha pasado a hechos concretos. La preservación del medio ambiente siempre estará ligada a la "tragedy of commons" (tragedia de los comunes) en el cual los intereses de una sola nación primara por encima de todos los involucrados. Como se ha visto desde 1999 con el Protocolo de Kyoto, en el cual China y Estados Unidos no ratifican el tratado pero si exigen cambios de modelo económico global o imponen la guerra contra el terrorismo, recursos que serían suficientes para preservar gran parte de la Amazonía Latinoamericana. 
Por otro lado, sin diversificación de economía e industria será muy difícil para países principalmente petroleros dejar de lado este modelo extractivista y este será el principal motivo por el cual el petróleo no se deje bajo tierra, lo que podría provocar mayores guerras civiles como las que suceden a diario en Nigeria o en su futuro guerras mundiales porque no se puede poner un gramo más de dióxido de carbono en la atmósfera. En el caso de la reactivación de la Iniciativa ITT varios colectivos ambientales, actores políticos y catedráticos mantienen que será necesario cambiar de gobierno en Ecuador para poder repensar la idea y evitar que se explote en su totalidad este Parque Nacional. Retomar el respeto a la Constitución del Ecuador que engloba los derechos a la naturaleza y la consulta previa, libre e informada, será lo que marque un cambio de pensamiento y reformule un desarrollo sustentable.

Los gobiernos seccionales de cada país deberán ser quienes tomen acciones concretas y mancomunadas dejando de lado ideologías políticas y preponderando la idea de un sistema ecológico estable, sano y sustentable que nos provea del oxígeno suficiente a largo plazo, sin necesidad de buscar nuevos planetas que sean habitables. Como dice el adagio "piensa de manera global y actúa de manera local". Otorgar derechos a la Naturaleza fue un paso importante para Ecuador que se debería replicar con el fin de salvar el planeta, conectar nuestra vida con las creencias indígenas que se enfoca directamente en el respeto a la Pacha Mama (Madre Tierra) y tener como bandera el conocer los ciclos vitales de los bosques para que se mantengan vivos.

Como mencionó Alicia Cahuiya, en la Sesión 256 de la Asamblea Nacional ${ }^{35}$ los pueblos indígenas pasan fronteras, no saben de límites imaginarios ni de qué territorio pueden transitar y cual no, para ellos la tierra es una sola y la utilizan para poder pernoctar, cazar y desarrollar su cultura.

Soy mujer waorani. Vengo de la Amazonía. Nosotros hemos sido conservacionistas. Queremos que respete el territorio. Pedimos territorio. Antes todo era inmenso. Cada vez los gobiernos están dividiendo. Nos están botando todo. Otra gente está administrando. Nosotros tenemos que administrar. No estamos de acuerdo con que nos den haciendo el trabajo. Gobierno decreta zona intangible, pero Taromenani no viven en un solo sitio. Pasan territorios Ecuador Perú. Necesitan un territorio binacional. Si no hubieran huaorani viviendo en la Amazonía. Porque mueren Taromenani-Huaorani por abrir carretera. Déjanos vivir como huaorani, esa es nuestra propuesta. Antes vivíamos bien, agua limpia, árbol sano. Donde tenemos huaoranis más pobreza estamos haciendo. Yo nací en río Nasinho. Qué hablan de Yasuní. Porque nosotros somos una mujer que hemos venido cuidando nuestra selva, nuestros árboles, nuestros ríos. Donde sale el petróleo, más hemos sido contaminados, más destruidos el territorio huaorani. (Alicia Cahuiya, Vicepresidenta de la Nacionalidad Huaorani del Ecuador)

La reactivación de la Iniciativa ITT preservará la vida y ayudará a crear una consciencia socio-ambiental de manera nacional para luego buscar la forma de impulsarla en los diferentes estamentos internacionales. Para algunos sectores de la sociedad civil será utópico pensar en la preservación del medio ambiente, mas a pesar del pesimismo que se haya podido demostrar por las actuaciones de los organismos internacionales, se cree firmemente que estos han logrado poner en debate estas nuevas problemáticas mundiales. Identificar las falencias de la Iniciativa ITT en su primera instancia será el paso inicial para poder reformular las estrategias e integrar de mejor manera a todos los actores, lo que podría llevar a su éxito.

\section{Conclusiones}

Para hablar de la riqueza del Parque Nacional Yasuní y la necesidad de apoyo internacional para preservarlo, tal vez podríamos asociarlo con el cuento de "El Lorax", un pequeño ser que hablaba por los árboles "porque los árboles no tienen lengua". Este personaje se le aparece al Once-ler, que, al encontrar el material preciso para hacer sus Thneed (especie de bufanda) comienza a talar todos los árboles para crear más de estos y así satisfacer su ambición. Hasta que no taló todos los árboles no entendió que este recurso era finito. Aún peor, apenas en ese momento fue cuando se dio cuenta de que ya no había animales, agua limpia y mucho menos oxígeno para respirar. Después de todo este daño El Lorax dejó al Once-ler una sola frase "A MENOS QUE", frase que entendería al pasar el tiempo y con la llegada de una persona que quiso saber que paso con el bosque y todo lo que había en él...A MENOS QUE alguien como tú se interese mucho más, nada va a cambiar. ${ }^{36}$ Si reemplazamos a los protagonistas con los actores de la Iniciativa ITT podríamos asociar la situación, hasta que no exista más petróleo no apreciaremos la riqueza que en realidad contenía la Reserva en la superficie.

Si bien la economía del Ecuador se sigue basando en la extracción de petróleo de la Amazonía sería necesario re-

35. Discurso dado en la Sesión 256 en la Asamblea Nacional del Ecuador. El video se encuentra a disposición en https://www.youtube.com/watch?$\mathrm{v}=$ oqqfiBCmxwI, fuente de esta cartera del Estado.

36. Cuentos de Dr. Seuss, "The Lorax" 
tomar la cuestión del costo-beneficio de la explotación de algunos bloques como los del ITT. Este tipo de crudo no llevará a obtener mayores réditos sin una refinería en funcionamiento. Adicionalmente no existen compañías que quieran invertir en estas actividades debido al alto costo de construcción y porque ya poseen estas empresas en otros países que tienen obra de mano más barata. Es por ello que el mantener el crudo en el subsuelo del oriente ecuatoriano hubiera sido una decisión sabia, aún sin la ayuda internacional el Estado se habría ahorrado millones de dólares si no perforaba más pozos.

Por otra parte, la Iniciativa Yasuní ITT fue un proyecto que Alberto Acota ha catalogado como "adelantada a su época" debido a que todavía no existe una real conciencia ambiental del caos que se vendrá en pocos años si se sigue desgastando la tierra de esta manera tan acelerada. En efecto los derechos de la naturaleza apenas están siendo reconocidos en algunos países y siguen siendo considerados una utopía de ambientalistas sentimentales. Aunque las comparaciones son odiosas, es necesario recordar que en la época colonial se consideraba que los afro descendientes no tenían alma por lo cual no podían tener derechos: el tiempo y el pensamiento evoluciona hacia respetar al otro. Es así que ahora decimos que la naturaleza no tiene derechos porque no habla, mas si todos habláramos por ella como en el cuento de Dr.Seuss, la realidad podría cambiar y adjudicar globalmente derechos a la naturaleza como un camino que nos conduzca a un mundo sustentable que nos permita alcanzar el Buen Vivir.

En el trasfondo, la Iniciativa ITT logró cimentar un debate internacional de cómo se manejan las agendas medio ambientales y cómo el mercado de carbono primará por encima de crear verdaderos cambios globales que mitiguen el calentamiento global. Lograr el compromiso de todas las naciones para reducir la comercialización de combustibles y derivados de petróleo podría ser la fórmula para alcanzar la reducción en la emisión de GEI y no solo hablar del éxito que está teniendo el Protocolo de Montreal en la disminución del agujero en la capa de ozono. De manera pesimista, llegará un punto en el cual estos componentes no se degradaran y será una suerte encontrar una solución a corto plazo para remediar el daño provocado en decenas de años.

No es objeto de este trabajo el recordar las convergencias y divergencias discursivas del Presidente Correa que llevó al fracaso de la Iniciativa ITT. Pero sí se puede considerar que un cambio de pensamiento y un nuevo actor social sería primordial para volver al debate de la preservación de la Reserva de la Biósfera Yasuní, no sólo porque su petróleo sea difícil de explotar, sino porque su verdadera riqueza está sobre la tierra. Se trata de un lugar mega di- verso, por tener especies de animales y plantas únicas en el mundo, que posee culturas tan antiguas como la tierra, que son capaces de enseñarnos cuál es el camino hacia una sociedad más respetuosa de lo que le rodea y un mundo en el cual la naturaleza sea la protagonista.

En suma, se ha podido observar que la Iniciativa ITT era el proyecto primordial para marcar el camino del cambio de la matriz productiva del Ecuador. Se ha constatado cómo protocolos, tratados y demás acciones conjuntas pueden ser llevadas a éxito si existen sanciones de por medio. Lastimosamente la negociación y la cara visible de este tipo de proyectos siempre serán esenciales al momento de tener reacciones positivas y apoyo de todas las esferas y niveles sociales. La integración de bloques regionales y la búsqueda de cooperación internacional que contengan metas, que identifiquen políticas a seguir y establezca sanciones reales, podrá guiar hacia un sistema socio-ambiental amigable que permita mitigar el calentamiento global y con eso evitar más catástrofes mundiales. 


\section{Bibliograffía}

ACOSTA A. GUDYNAS E. MARTÍNEZ E. \&VOGEL J. (2010) "Dejar el crudo en tierra o la búsqueda del paraíso perdido" ACOSTA, A. y MARTÍNEZ, E.ITT-Yasuní entre el petróleo y la vida; AbyaYala, Quito.

ACCIÓN ECOLÓGICA (S/F) Deuda Ecológica. Consultado el 24/02/2017. Disponible en: http://www.accionecologica.org/deuda-ecologica.

ASAMBLEA NACIONAL DEL ECUADOR (2013) Asamblea Aprobó Declaratoria de Interés Nacional la Explotación en el Parque Nacional Yasuní. Consultado el 24/02/2017. Disponible en: <http://www. asambleanacional.gob.ec/es/contenido/asamblea_aprobo_declaratoria_de_interes_nacional_la_explotacion_en_el_parque_nacional>

ÁVILA SANTAMARÍA R. (2010) El derecho de la naturaleza: fundamentos. Universidad Andina Simón Bolivar. Quito-Ecuador

ÁVILA SANTAMARÍA R. (2015) "El Yasuní, los "modelos de desarrollo" y el sumakKawsay desde las mujeres indígenas". BALSECA F. \& MONTÚFAR C. Ecuador desafíos para el presente y el futuro. Ediciones Tierra. Universidad Andina Simón Bolivar. Quito-Ecuador.

BARRETT S. (2000) "Montreal contra Kyoto: la cooperación internacional y el medio ambiente mundial." Grunberg I., Kaul I., Stern M. Bienes Públicos Mundiales: La cooperación internacional en el siglo XXI. Oxford University.

BIROH, F. (2016) The World Needs a Stable Oil Market. "Deutsche Welle" Consultado el 25/02/2017. Disponible en: <https://www.regjeringen. no/en/aktuelt/the-world-needs-a-stable-oil-market/id2523498/>

DE SOUZA, B. (2009) “De Copenhague a Yasuní”. ACOSTA, A. y MARTÍNEZ, E.ITT-Yasuní entre el petróleo y la vida; AbyaYala, Quito.

CABODEVILlA, M. (2016) Los Huaorani en la Historia de los Pueblos del Oriente. 3ra Edición. Quito-Ecuador.

CONSTITUCIÓN DE LA REPÚBLICA DEL ECUADOR (2008) Publicación Oficial de la Asamblea Nacional del Ecuador. Montecristi.

CORREA, R. (2007) Discurso pronunciado ante el Foro de Presidentes sobre el Cambio Climáticos de las Naciones Unidas. Nueva York. Consultado 10 de julio $2016<$ www.presidencia.gob.ec $>$

COOK L. \&SACHS J. (2000) "Los bienes públicos regionales en la ayuda internacional". GRUNBERG I., Kaul I., STERN M. Bienes Públicos Mundiales: La cooperación internacional en el siglo XXI. Oxford University.

DILGER, G. (2010) “Guacamayos en vez de torres de petróleo”. ACOSTA A. y MARTÍNEZ E.ITT-Yasuní entre el petróleo y la vida; AbyaYala, Quito.
DW (2010) GermanyVoicesreservationsaboutEcuador'soilfund.Consultado el 19 de Junio de 2016. <http://www.dw.com/en/germany-voices-reservations-about-ecuadors-oil-fund/a-6039547>

FINER M., VIJAY V., PONCE F., JENKINS C. \&KAHN T. (2010) "La Reserva de la Biósfera Yasuní de Ecuador: una breve historia moderna y los retos de la conservación" ACOSTA A. y MARTÍNEZ E.ITT-Yasuní entre el petróleo y la vida;AbyaYala, Quito.

HEAL G. (2000) "Nuevas estrategia para la provisión de bienes públicos mundiales: Como aprender de los desafíos ambientales internacionales"GRUNBERG I., KAUL I., STERN M. Bienes Públicos Mundiales: La cooperación internacional en el siglo XXI.Oxford University.

HUMPHREY M. (2009) “Climate Change”.McLEAN I. \&McMILLAN A. Concisedictionary of Politics.Oxford University

JAYARAMAN R. \& KANBUR R. (2000) “Los bienes públicos internacionales y el argumento en favor de la ayuda externa" GRUNBERG I., KAUL I., STERN M. Bienes Públicos Mundiales: La cooperación internacional en el siglo XXI. Oxford University.

KAHHAT F. (2013) Las industrias extractivistas y sus implicaciones políticas y económicas. No. 174, Instituto de Estudios Internacionales - Universidad de Chile. Santiago

LARREA, C. (2009) YASUNÍ ITT: Una Iniciativa para Cambiar la Historia, UNDP Ecuador, Cooperación Ecuador - República Federal de Alemania, Fondo para el Logro de los ODM.

LARREA C. (2010) "Por qué el Ecuador debe mantener el petróleo del ITT bajo tierra" Acosta Alberto y Martínez Esperanza ITT-Yasuní entre el petróleo y la vida; AbyaYala, Quito.

MATT, F., VIJAY, V., PONCE F., JENKINS, C. \&KAHN, T. (2010) "La Reserva de la Biósfera Yasuní de Ecuador: una breve historia moderna y los retos de la conservación".ACOSTA, A. y MARTÍNEZ, E.ITT-Yasuní entre el petróleo y la vida; AbyaYala, Quito.

MENDOZA M., PÉREZ V. (2010) Energías renovables y movimientos sociales en América Latina. No.165 Instituto de Estudios Internacionales - Universidad de Chile. Santiago

MOVIMIENTO MUNDIAL POR LOS BOSQUES TROPICALES (2009) "Nigeria: petróleo bajo tierra y mucho más" Boletín No. 149 Disponible en: <http://wrm.org.uy/oldsite/boletin/149/Nigeria.html>

ORGANIZACIÓN DE LAS NACIONES UNIDAS (2000) Objetivos de Desarrollo del Milenio. Consultado el 05/06/2016, Disponible en: $<$ http://www.un.org/es/millenniumgoals/mdgnews.shtml > 
ORGANIZACIÓN DEL TRATADO DE COOPERACIÓN AMAZÓNICA (1978) "Tratado de Cooperación Amazónica". Consultado el 25/06/2016. Disponible en: <http://otca.info/portal/admin/_upload/ tratado/TRATADO_COOPERACION_AMAZONICA_ESP.pdf >

PRESIDENCIA DE LA REPÚBLICA DEL ECUADOR (2007) Discurso Del Presidente En La 62 Asamblea General De Las Naciones Unidas, En Nueva York. Consultado el 25/06/2016. Disponible en: <http:// www.presidencia.gob.ec/discursos/>

PROGRAMA DE LAS NACIONES UNIDAS PARA EL DESARROLLO (2011) Fondo de Fideicomiso: Manual de Procedimiento para el Funcionamiento del Comité de Dirección Ecuador Yasuní ITT (2011). Quito-Ecuador.

PROTOCOLO DE KYOTO DE LA CONVENCIÓN MARCO DE LAS NACIONAES UNIDAS SOBRE EL CAMBIO CLIMÁTICO (1998) Naciones Unidas. Consultado el 23/06/2016. Disponible en: <www. un.org>

SEVILLA R. (2010) "Cómo transformar una idea y un ideal en una iniciativa práctica y ejecutable” ACOSTA A. y MARTÍNEZ E.ITT-Yasuní entre el petróleo y la vida; AbyaYala, Quito.

SOLIZ M. (2015) "Extractivismo, soberanía y salud" BALSECA F.\&MONTÚFAR C. Ecuador desafíos para el presente y el futuro. Ediciones Tierra. Universidad Simón Bolivar. Quito-Ecuador.

SOLOMÓN S., IVY D., KINNISON D., MILLS M., NEELY R., SCHMIDT A. (2016) "Emergence of healing in theAntarctic ozone layer" ScienceMagazine.Vol. 353. Issue 6269, pp. 269-274. Consultado el 01/06/2016. Disponible en: <http://science.sciencemag.org/content/353/6296/269.full>

SWING K. (2013) If Ecuador must drill for Yasuní oil, let's encourage the least damaging methods. Consultado el 01/06/2016. Disponible en:<www.theguardian.com/environment/2013/avg/28/ecuador-drill-oil-yasuni-damage $>$

UNITED NATIONS EDUCATIONAL, SCIENTIFIC ANDA CULTURAL ORGANIZATION (S/F) BiosphereReserves.Consultado el 27/02/17. Disponible en: <http://www.unesco.org/new/en/natural-sciences/environment/ecological-sciences/biosphere-reserves/>

UNITED NATIONS FRAMEWORK CONVENTION ON CLIMATE CHANGE (S/F) Comunicado de Prensa: El Protocolo de Kyoto entrará en vigor el 16 de Febrero de 2005. Consultado el 27/02/2017. Disponible en:<https://unfccc.int/files/press/news_room/press_releases_and_advisories/application/pdf/press041118_esp.pdf>

UNIVERSIDAD SAN FRANCISCO DE QUITO (1994) Tiputini. Consultado el 24/02/2017. Disponible en:<https://www.usfq.edu.ec/programas_academicos/Tiputini/Paginas/About-us.aspx>
VILLAVICENCIO F. (2010) “¿Integración o privatización? Entrega de Sacha a PDVSA"ARAÚZ A., SALTOS N., VILLAVICENCIO F. y ZURITA C. El Discreto Encanto de la Revolución Ciudadana, 2da Edición, Quito-Ecuador.

VOGLER J. (2014) "Environmental Issues"BAYLIS J., SMITH S., \& OWENS P.The Globalization of World Politics: An introduction to international relations. Oxford University

WORLD GOVERNANCE (S/F) Bienes Comunes Mundiales. Consultado el 24/02/2017. Disponible en: <http://www.world-governance. org/article1193.html> 